\title{
从三氟甲基化反应的近年进展看有机氟化学的发展趋势
}

\author{
卿凤悀 $a, b$ \\ ( ${ }^{a}$ 中国科学院上海有机化学研究所 有机氟化学重点实验室 上海 200032) \\ ( ${ }^{b}$ 东华大学化学化工与生物工程学院 上海 201620)
}

\begin{abstract}
摘要 由于含三氟甲基的化合物在医药、农药等领域得到广泛应用, 发展向有机分子引入三氟甲基的方法成为当前的 热点研究课题. 分别总结了国外和我国学者近年(主要是 2009 2011 年)在三氟甲基化反应研究领域取得的研究成果. 从近年三氟甲基化反应的研究进展, 作者提出一些值得关注的有机氟化学发展方向.

关键词 三氟甲基化; 有机氟化学; 进展

\section{Recent Advances of Trifluoromethylation}

\author{
Qing, Fengling ${ }^{a, b}$ \\ ( ${ }^{a}$ Key Laboratory of Organofluorine Chemistry, Shanghai Institute of Organic Chemistry, Chinese Academy of \\ Sciences, Shanghai 200032) \\ $\left({ }^{b}\right.$ College of Chemistry, Chemical Engineering and Biotechnology, Donghua University, Shanghai 201620)
}

\begin{abstract}
Many biologically active compounds contain $\mathrm{CF}_{3}$ group as the essential motif. As a result, much attention has been paid to the development of new synthetic methods for the introduction of $\mathrm{CF}_{3}$ group into diverse organic compounds. This review takes a critical look at recent advances of trifluoromethyltion reactions.

Keywords trifluoromethylation; organofluorine chemistry; advance
\end{abstract}

1896 年 Swarts 等有关一氟乙酸乙酯的合成揭开了 有机氟化学的序幕. 在这百余批中, 几乎全是人工合成 的含氟有机化合物(含氟高分子材料)已在国防工业、高 新技术产业和生命科学中发挥越来越重要的作用. 据统 计, 目前 $20 \%$ 的医药和 30\%农药中至少含有一个氟原 子 $^{[1]}$. 就有机氟化学学科而言, 它已经成为有机化学的 重要分支, 无数事实证明它既服从有机化学的共同规 律, 又以碳氟键的特点丰富和发展了有机化学. 从有机 氟化学学科发展的历史来看, 20 世纪 30 年代到 50 年代 含氟致冷剂的研制、聚四氟乙烯的发现和美国曼哈顿工 程的实施等极大地推动了有机氟化学的发展. 近年来, 生物医学研究、治疗和诊断对含氟有机化合物的需求及 金属有机化学在含氟有机化合物合成中的广泛应用使 有机氟化学学科处于发展的黄金时期. 在庆祝 2011 年 国际化学年时, Nature 首次发表了一篇关于含氟有机化 合物合成的综述文章 ${ }^{[2]}, 2011$ 年 Science 罕见地发表了 4
篇有机氟化学研究论文 ${ }^{[3]}$. 表明有机氟化学目前已是有 机化学的热点研究领域. 在此笔者将根据自己的研究兴 趣选择三氟甲基化反应的近年进展来说明有机氟化学 的发展趋势. 本文将分为三个部分: 三氟甲基化反应进 展、我国学者在三氟甲基化反应的代表性工作、有机氟 化学发展趋势.

\section{1 三氟甲基化反应进展}

由于三氟甲基 $\left(\mathrm{CF}_{3}\right)$ 具有强吸电子性、亲脂性和稳定 的 $\mathrm{C}-\mathrm{F}$ 键等特性, 将其引入到有机化合物中能够显著 改变化合物的酸性、偶极距、极性、亲脂性以及其化学 和代谢稳定性. 因此含三氟甲基的化合物已在医药、农 药和材料等领域得到广泛应用. 如治疗精神抑郁的药物 Prozac、治疗关节炎的药物 Celebrex 和治疗 II 型糖尿病 的药物 Januvia 等都含有三氟甲基(图 1).

\footnotetext{
*E-mail: flq@mail.sioc.ac.cn

Received February 2, 2012; revised March 1, 2012; published online April 10, 2012. 2012CB21600).

国家自然科学基金(Nos. 21072028, 20832008)和国家重点基础研究发展计划(No. 2012CB21600)资助项目.
}

Project supported by the National Natural Science Foundation of China (Nos. 21072028, 20832008) and the National Basic Research Program of China (No. 
<smiles>CNCCC(Oc1ccc(C(F)(F)F)cc1)c1ccccc1</smiles>

Prozac

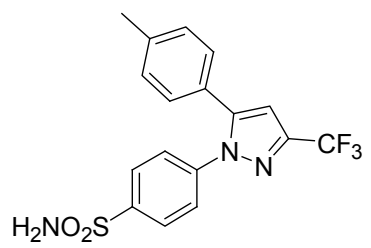

Celebrex<smiles>N[C@@H](CC(=O)N1CCn2c(nnc2C(F)(F)F)C1)Cc1cc(F)c(F)cc1F</smiles>

图 1 含三氟甲基的代表药物

Figure 1 Drugs containing trifluoromethyl group

几十年来, 化学家发展了各种不同类型的向有机分 子引入三氟甲基的反应, 但这些方法存在反应条件苛 刻、原料难得及选择差等缺点. 近几年来, 在金属有机 化学研究的推动下, 三氟甲基化反应取得了重大突破 ${ }^{[4]}$. 同时，对经典的通过三氟甲基自由基的反应开展了新的 探索. 本部分将根据反应类型来论述近年发展的三氟甲 基化反应. 我国学者在该领域的研究成果将在本文的第 二部分总结. 必须说明的是: 本文论述的三氟甲基化反 应是指使用三氟甲基化试剂经过一步反应将三氟甲基 $\left(\mathrm{CF}_{3}\right)$ 引入到有机分子.

\section{1 通过三氟甲基自由基 $\left(\mathrm{CF}_{3} \cdot\right)^{\cdot}$ 的三氟甲基化反应}

产生三氟甲基自由基(三氟甲基自由基前体)的方法 和它对芳香化合物的三氟甲基化反应已经研究了几十 年. 2011 年美国的二个课题组进一步探索了三氟甲基自 由基 $\left(\mathrm{CF}_{3} \cdot\right)$ 对芳香和芳香杂环的三氟甲基化反应. 他们 发展的三氟甲基化反应能在非常温和的反应条件下进 行. 应用这些方法可对目前已上市的药物分子进行后三 氟甲基化反应(late stage trifluoromethylation)来合成一系 列含三氟甲基的类药物分子, 为发现含氟药物提供方便 和有效的方法.

美国 Scripps 研究所的 Baran 小组 ${ }^{[5]}$ 发现以 $\mathrm{CF}_{3} \mathrm{SO}_{2} \mathrm{Na}$ 作为三氟甲基源, 通过自由基机理实现了芳 香杂环的三氟甲基化反应(Scheme 1). 该反应可在室温 下进行, 不需要使用催化剂, 有很好的官能团兼容性. 作者已将该反应应用于对 Caffeine 和 Varenicline 等药物 的三氟甲基化反应. 必须指出的是：法国氟化学家 Langlois 等 ${ }^{[6]}$ 已在 20 世纪 90 年代报道了 $\mathrm{CF}_{3} \mathrm{SO}_{2} \mathrm{Na}$ 在 氧化剂存在下对富电子芳香化合物的三氟甲基化反应.

2011 年, 美国 Princeton 大学的 MacMillan 等 ${ }^{[7]}$ 在 Nature 上发表了类似的研究工作. 他们使用 $\mathrm{CF}_{3} \mathrm{SO}_{2} \mathrm{Cl}$ 作为三氟甲基源, 以 $\mathrm{Ru}(\mathrm{phen})_{3}^{2+}$ 为光催化剂, 在家用灯
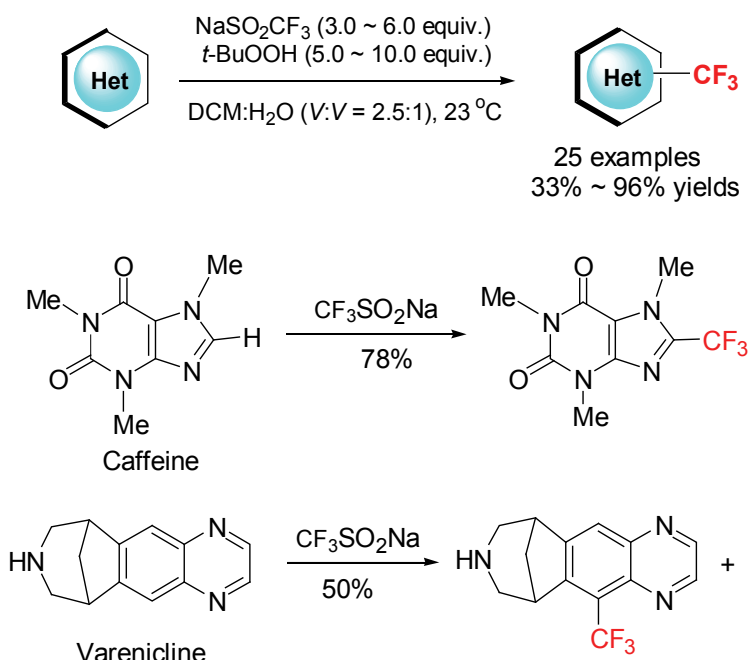<smiles>FC(F)(F)c1cnc2cc3c(cc2n1)C1CNCC3C1</smiles>

Scheme 1

泡的光照下, 实现了对芳烃与杂环芳烃的三氟甲基化反 应(Scheme 2). 五元杂环、六元杂环以及未活化的芳烃 都能顺利实现三氟甲基化. 该温和的反应条件可直接对 药物分子(Ibuprofen, Lidocaine, Lipitor 等)进行三氟甲基 化. 同样需要指出的是: 日本氟化学家已在 20 世纪 90 年代报道了在金属络合物的催化下, $\mathrm{CF}_{3} \mathrm{SO}_{2} \mathrm{Cl}$ 对芳香化 合物的三氟甲基化反应 ${ }^{[8 a]}$ ，中国科学院上海有机化学研 究所黄维垣教授在 1992 年报道了 $\mathrm{CF}_{3} \mathrm{SO}_{2} \mathrm{Br}$ 与烯烃发生 自由基加成反应 ${ }^{[8 b]}$.
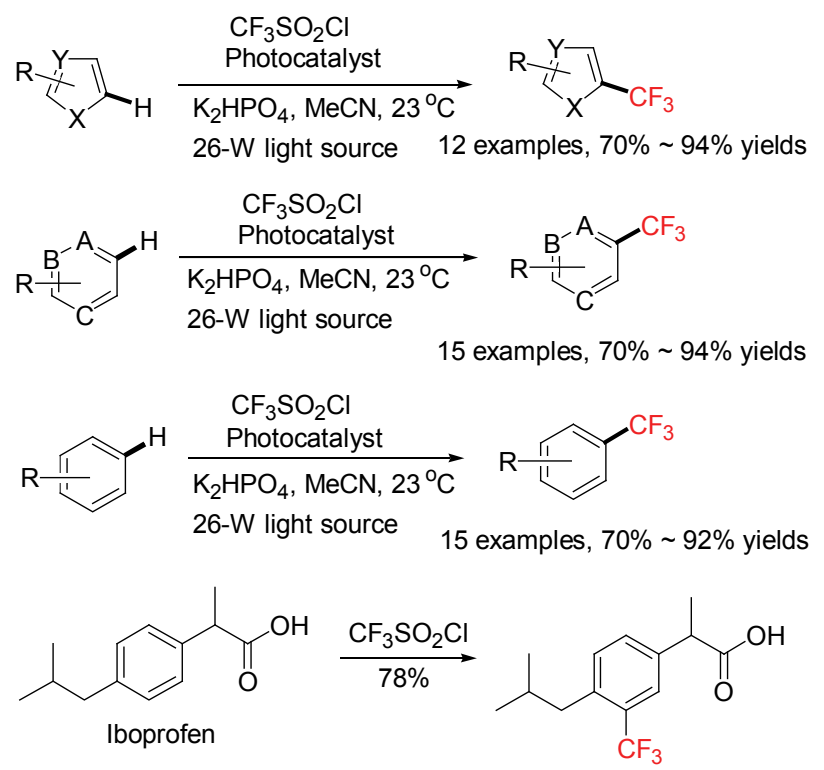

Scheme 2 


\section{2 钯参与(催化)的三氟甲基化反应}

钯催化的偶联反应现在已成为形成碳一碳键的常用 方法. Heck, Negishi 和 Suzuki 三人由于在钯催化的偶联 反应做出的突破贡献获得了 2010 年诺贝尔化学奖. 但 关于钯催化形成 $\mathrm{C}-\mathrm{CF}_{3}$ 的反应一直困扰着化学家, 这 主要是由于 $\mathrm{L}_{n} \mathrm{PdArCF}_{3}$ 很难发生还原消除反应. Hartwig 等 ${ }^{[9]}$ 在 2004 年发现(dppz) $\mathrm{Pd}\left(o\right.$-tol) $-\mathrm{CF}_{3}$ 在 $130{ }^{\circ} \mathrm{C}$ 下加热 数天不发生变化, $(\mathrm{dppz}) \mathrm{Pd}(o-$ tol $)-\mathrm{CH}_{2} \mathrm{CF}_{3}$ 在 $110{ }^{\circ} \mathrm{C}$ 下 加热 $36 \mathrm{~h}$ 以 $96 \%$ 的产率得到 $\mathrm{ArCH}_{2} \mathrm{CF}_{3}$, 而 $\mathrm{L}_{n} \mathrm{PdArCF}_{3}$ 在 $40{ }^{\circ} \mathrm{C}$ 下反应 $4 \mathrm{~h}$ 以 $99 \%$ 的产率得到 $\mathrm{ArCH}_{3}$ (Scheme 3).

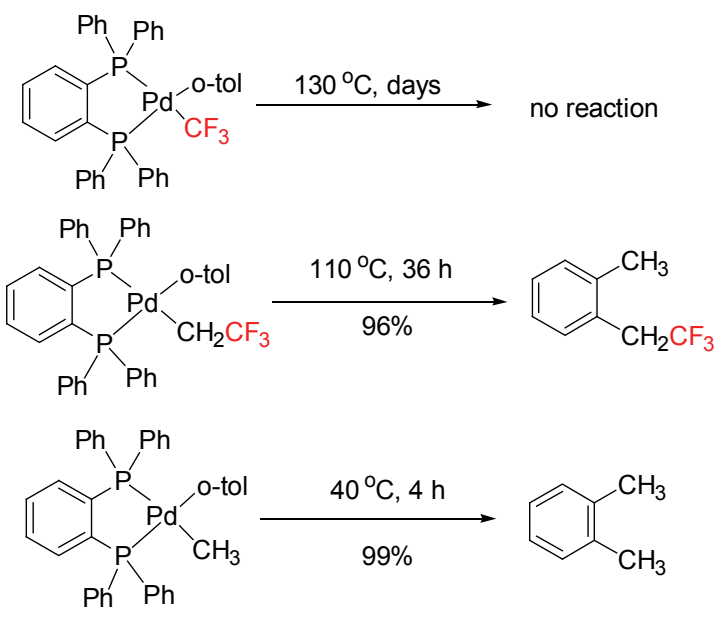

Scheme 3

2006 年, 在解决 $\mathrm{L}_{n} \mathrm{PdArCF}_{3}$ 很难发生还原消除反应 这一难题上取得突破. Grushin 等 $^{[10]}$ 合成了一系列含不 同配体 $\mathrm{L}_{n} \mathrm{PdArCF}_{3}$ 络合物, 当用空间位阻大的 Xantphos 为配体得到了含三氟甲基二价钯络合物 [(xantphos)$\left.\mathrm{PdPhCF}_{3}\right]$, 该络合物在 $80{ }^{\circ} \mathrm{C}$ 反应 $3 \mathrm{~h}$ 几乎能定量的得 到三氟甲基苯(Eq. 1). 这是第一例含三氟甲基二价钯络 合物可进行还原消除反应形成 $\mathrm{CF}_{3}-\mathrm{C}$ 键.

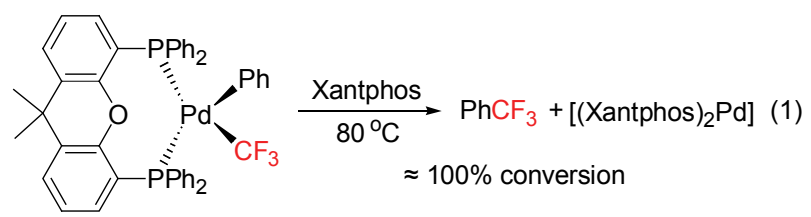

Buchwald 等 ${ }^{[11]}$ 在 2009 年首次实现从 $\mathrm{Pd}^{\mathrm{II}}-\mathrm{F}$ 络合 物上还原消除得到芳基氟化合物的基础上, 2010 年报道 了钯催化的三氟甲基化反应. 通过使用 BrettPhos 和 $\mathrm{RuPhos}$ 为配体, 以 $\mathrm{Et}_{3} \mathrm{SiCF}_{3}$ 为三氟甲基源, 第一次实现 了 $\operatorname{Pd}(0)$ 催化的芳基氯化物的三氟甲基化, 该反应也是 通过含三氟甲基的二价钯络合物的还原消除反应进行 的(Scheme 4) ${ }^{[12]}$. 该反应成功的关键在于选择大体积的 单齿膦配体 BrettPhos. 但 Buchwald 教授在文章中也指
出该反应有一些不足之处：反应温度高 $\left(130 \sim 140{ }^{\circ} \mathrm{C}\right)$, 三氟甲基化试剂贵 $\left(\mathrm{CF}_{3} \mathrm{SiEt}_{3}\right)$ 和带有羰基的芳基氯化物 不能将三氟甲基直接引入到芳环上. 最近, Buchwald 等 ${ }^{[13]}$ 发现使用 $t$-BuXPhos 作配体，以 $\mathrm{TMSCF}_{3}$ 和 $\mathrm{Et}_{3} \mathrm{SiCF}_{3}$ 为三氟甲基源, 完成了钯催化下烯基磺酸酯的 三氟甲基化(Scheme 4).
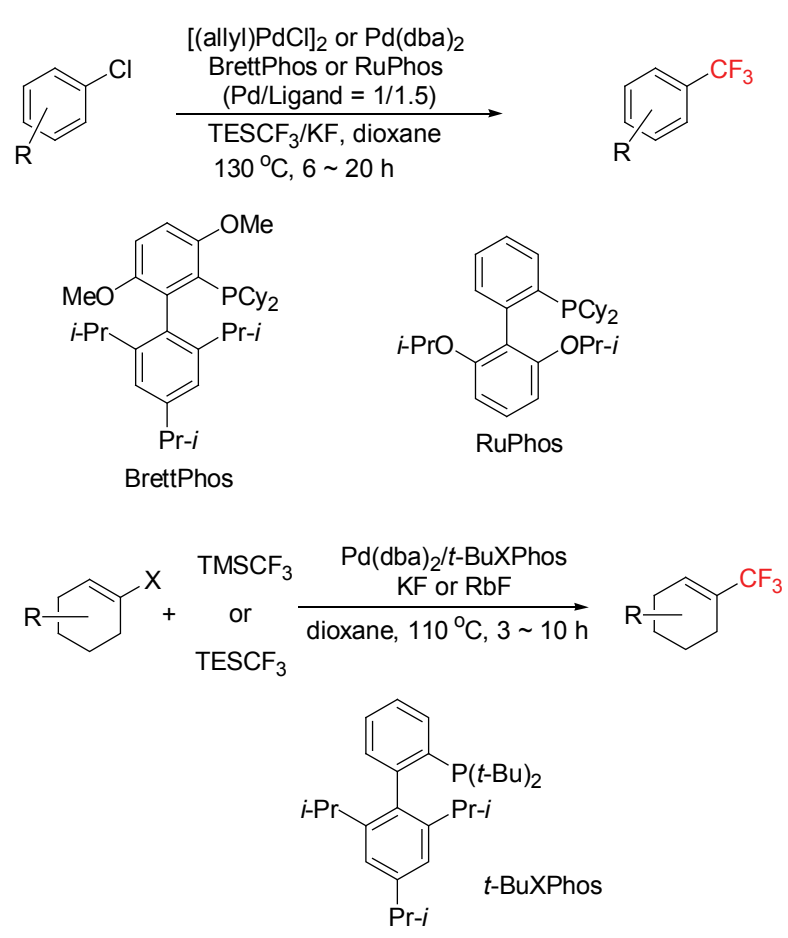

Scheme 4

Sanford 课题组 ${ }^{[14]}$ 在系统研究 $\operatorname{Pd}(\mathrm{IV})$ 络合物后, 发 现 Pd(IV)络合物比 Pd(II)络合物更易进行还原消除反应. 因此，她设想能否通过含三氟甲基四价钯络合物 $\left(\mathrm{LnPd}^{\mathrm{IV}} \mathrm{ArCF}_{3}\right)$ 的还原消除反应来合成 $\mathrm{Ar}-\mathrm{CF}_{3}$. 在室温 下使用含氟氮键的亲电氟化试剂将含三氟甲基的二价 钯络合物氧化为四价钯络合物, 该含三氟甲基的四价钯 络合物在 $80{ }^{\circ} \mathrm{C}$ 下发生还原消除反应得到 $\mathrm{Ar}-\mathrm{CF}_{3}$ (Scheme 5). Sanford 课题组分离出含三氟甲基的四价钯 络合物, 并通过 X-ray 确定了它的结构.

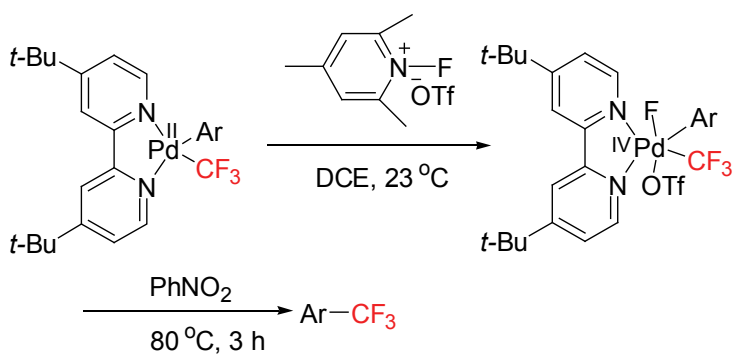

\section{Scheme 5}

$\mathrm{Yu}$ 等 ${ }^{[15]}$ 报道了钯催化下芳环的亲电三氟甲基化反 应(Eq. 2). 该反应使用亲电的三氟甲基化试剂作为三氟 
甲基源. 但该反应有局限性：芳环 $\mathrm{C}-\mathrm{H}$ 的邻位必须有 导向基团.<smiles>[R]c1ccc(-c2ccccn2)cc1</smiles>

$\mathrm{Pd}(\mathrm{OAc})_{2}$ (0.1 equiv.), $\mathrm{Cu}(\mathrm{OAc})_{2}$ (1.0 equiv.) $\left[\mathrm{R}_{2} \mathrm{SCF}_{3}\right]\left[\mathrm{BF}_{4}\right]$ (1.5 equiv.), TFA (10 equiv.) DCE, $110^{\circ} \mathrm{C}, 48 \mathrm{~h}$<smiles>[R]c1ccc(-c2ccccn2)c(C(F)(F)F)c1</smiles>

\section{3 铜参与(催化)的三氟甲基化反应}

自从 Mcloughlin 和 Thrower ${ }^{[16]}$, Kobayashi 和 Kumadaki ${ }^{[17]}$ 在 1969 年分别报道在铜粉存在下, $\mathrm{CF}_{3} \mathrm{I}$ 与 芳基碘反应生成三氟甲基芳基化合物后，铜参与的三氟 甲基化反应一直是合成含三氟甲基化合物最常用的方 法，一系列的三氟甲基化试剂被发现 ${ }^{[4]}$. 1986 年 Burton 等 ${ }^{[18]}$ 在研究铜参与的三氟甲基化反应时, 通过 ${ }^{19} \mathrm{~F} \mathrm{NMR}$ 第一次发现该反应是通过 $\mathrm{CuCF}_{3}$ 络合物进行. 2008 年, Vicic 等 ${ }^{[19]}$ 首次报道了氮杂卡宾配体 $(\mathrm{NHC})$ 稳定 $\mathrm{CuCF}_{3}$ 的络合物 $(\mathrm{NHC}) \mathrm{CuCF}_{3}$. 氮杂卡宾铜络合物 $(\mathrm{NHC}) \mathrm{Cu}$ 与 $\mathrm{CF}_{3} \mathrm{TMS}$ 反应生成( $\left.\mathrm{NHC}\right) \mathrm{CuCF}_{3}$ (Eq. 3). ( $\mathrm{NHC}^{\mathrm{CuCF}}$ 能 够分离, 且在室温下能稳定存在, 可通过 X-ray 确定它 的结构.
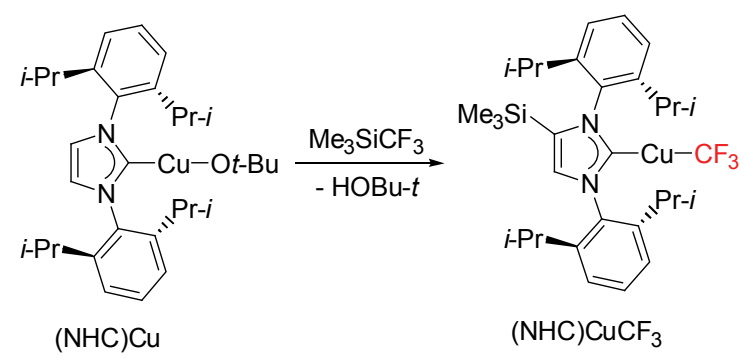

在上面工作的基础上, Vicic 等 ${ }^{[19]}$ 选用饱和的氮杂 卡宾铜络合物 $(\mathrm{NHC}) \mathrm{Cu}$ 与 $\mathrm{CF}_{3} \mathrm{TMS}$ 反应生成三甲基硅 基没有插入咪唑环的( $\mathrm{SIPr}-i) \mathrm{CuCF}_{3}$ (Scheme 6). 该络合 物结构也通过 X-ray 得到确定. 但是, $(\mathrm{SIPr}-i) \mathrm{CuCF}_{3}$ 在空 气中很不稳定, 并且发现通过原位生成的 $(\mathrm{SIPr}-i) \mathrm{CuCF}_{3}$ 与芳基碘化物反应以几乎定量的产率得到三氟甲基芳 基化合物(Scheme 6).

2011 年, Hartwig 等 ${ }^{[20]}$ 报道了在室温下 $[\mathrm{CuOBu}-t]_{4}$ 先与 1,10-phenantholine (phen) 反应再与 $\mathrm{CF}_{3} \mathrm{TMS}$ 反应, 以 $96 \%$ 的分离产率得到桔红色固体 [(phen) $\left.\mathrm{CuCF}_{3}\right]$ (Scheme 7). [(phen) $\mathrm{CuCF}_{3}$ ] 在室温和氮气下保存一个月 不会分解. [(phen) $\mathrm{CuCF}_{3}$ 与芳基碘反应以几乎定量的产 率得到三氟甲基芳基化合物(Scheme 7).
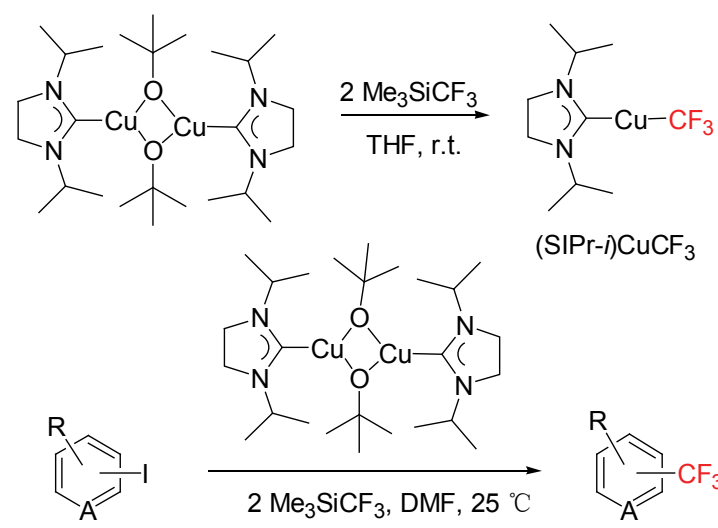

$(\mathrm{SIPr}-i) \mathrm{CuCF}_{3}$

\section{Scheme 6}

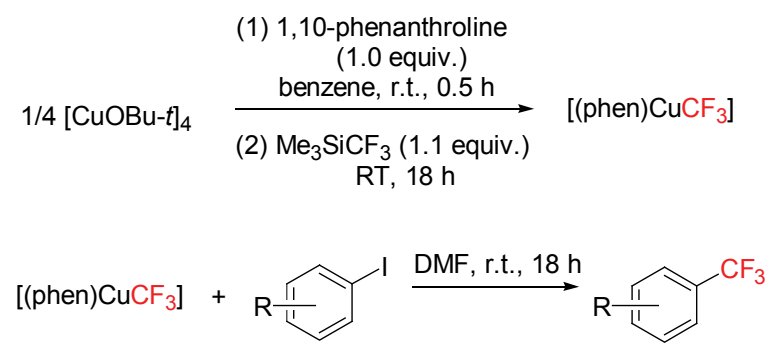

Scheme 7

Hartwig 等[21]不久后报道了使用 $\left[(\right.$ phen $\left.) \mathrm{CuCF}_{3}\right]$ 将三 氟甲基引入到 1,3-二取代芳烃中. 该方法是通过二步反 应完成的：首先 1,3-二取代芳烃在 Ir 的催化下生成相应 的芳基硼酸酯. 该芳基硣酸酯不需要分离纯化，然后在 空气存在下与 $\left[(\right.$ phen $\left.) \mathrm{CuCF}_{3}\right]$ 反应得到三氟甲基化合物， 但产物的分离产率仅为中等(Eq. 4).

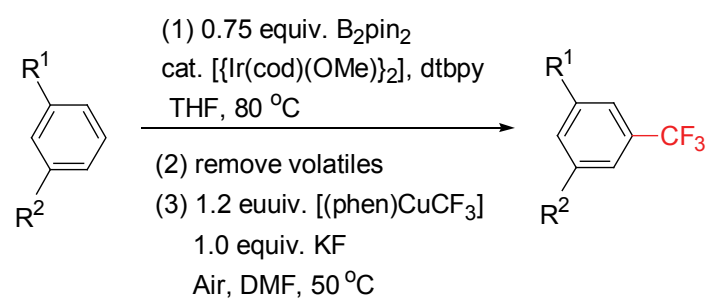

2011 年, Grushin 等 ${ }^{[22]}$ 合成出三苯基膦配体稳定的 三氟甲基铜试剂 $\left[\left(\mathrm{Ph}_{3} \mathrm{P}\right)_{3} \mathrm{Cu}\left(\mathrm{CF}_{3}\right)\right]$. 该络合物在配体 $t$-Bu-bpy 存在下对芳基碘的三氟甲基化反应产率可达到 $55 \% \sim 90 \%$ (Scheme 8).

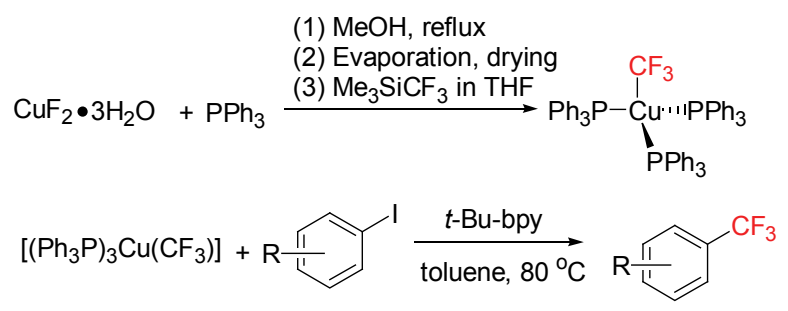

Scheme 8 
日本学者 Amii 等 ${ }^{[23]}$ 较系统地研究了铜参与下的芳 基三氟甲基化反应. 他们发现在反应体系中加入与 $\mathrm{Cu}(\mathrm{I})$ 形成络合物的二胺配体可实现铜催化的三氟甲基 化反应(Eq. 5). 但该反应条件仅适用于吸电子基团取代 的芳基碘化物. 这是三氟甲基化反应领域的一大突破, 因为除陈庆云教授等在 1989 年报道过铜催化的三氟甲 基化反应外(见本文的第二大部分), 铜参与的三氟甲基 化反应都是使用当量的铜.
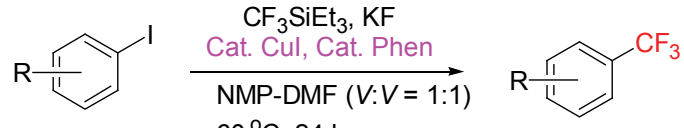

$60^{\circ} \mathrm{C}, 24 \mathrm{~h}$

在三氟甲基化试剂方面, 近年也有一些发展. 由于 现在已商品化的大部分三氟甲基化试剂是易挥发的液 体 (如: $\mathrm{CF}_{3} \mathrm{TMS}$ 沸点: $55{ }^{\circ} \mathrm{C}$ ), 因此德国学者 Roschenthaler 和 Gooßen 等 ${ }^{[24]}$ 从 $\mathrm{CF}_{3} \mathrm{TMS}$ 合成出 $\mathrm{CF}_{3} \mathrm{~B}(\mathrm{OMe})_{4} \mathrm{~K}$. 该化合物在空气中是稳定的固体. 他们 发现在铜催化下 $\mathrm{CF}_{3} \mathrm{~B}(\mathrm{OMe})_{4} \mathrm{~K}$ 对芳基碘的三氟甲基化 反应 ${ }^{[24]}$ 和对芳基嗍酸酯的氧化三氟甲基化反应(Scheme 9) ${ }^{[25]}$.

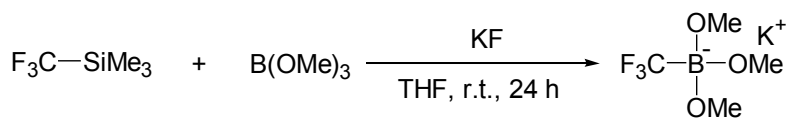

$$
\begin{aligned}
& \text { + }
\end{aligned}
$$

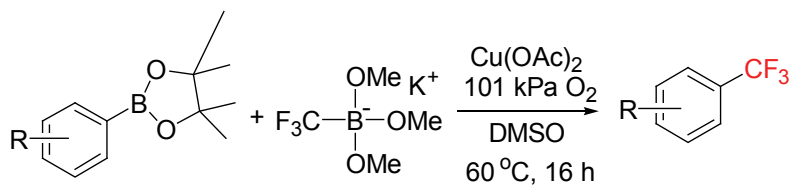

Scheme 9

在钯参与(催化)和铜参与(催化)的三氟甲基化反应 中, 都采用芳基(烯基)卤化物(主要是碘化物)作为反应 底物. 自 2010 年卿风领课题组开始探索芳基硼酸的氧 化三氟甲基化反应后(见本文的第二大部分), 芳基硼酸 与亲电三氟甲基化试剂的反应(见本文的第二大部分)及 与亲核三氟甲基化试剂的氧化三氟甲基化反应相继被 报道 ${ }^{[20,25,26]}$. Buchwald 等 ${ }^{[26]}$ 采用氧气为氧化剂, 在室温 下实现了芳基硼酸的氧化三氟甲基化反应(Eq. 6).

$$
\begin{gathered}
\mathrm{CsF}, \mathrm{O}_{2}, \mathrm{DCE} \\
4 \mathrm{AS} \text {, r.t. }
\end{gathered}
$$

近二年来在 $\mathrm{C}-\mathrm{H}$ 键的直接三氟甲基化反应取得重 大突破, 我国学者在该领域做出重要贡献(见本文的第
二大部分). 2011 年, Buchwald 等 ${ }^{[27]}$ 发现在 $\left[(\mathrm{MeCN})_{4} \mathrm{Cu}\right]-$ $\mathrm{PF}_{6}$ 的催化下, 亲电三氟甲基化 Togni 试剂与未活化的 烯烃反应可生成三氟甲基化的烯丙基化合物，该反应条 件相当温和, 产物位置选择性好, 但该反应条件不能应 用于环烯(Eq. 7).

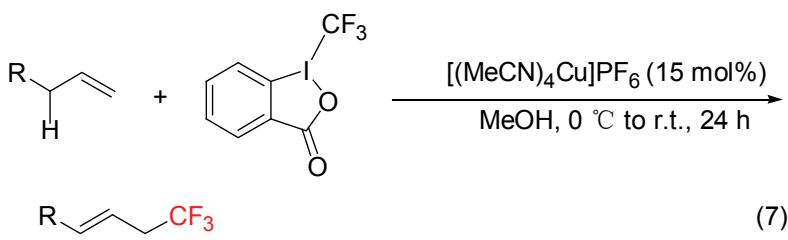

\section{4 不对称三氟甲基化反应}

美国 Princeton 大学的 MacMillan 小组在羰基 $\alpha$-位 的不对称三氟甲基化反应取得突破性的研究成果. 2009 年他们 ${ }^{[28]}$ 发现使用 $\mathrm{CF}_{3} \mathrm{I}$ 为三氟甲基源在有机小分 子催化剂和光催化剂催化下, 能将三氟甲基不对称引入 到醛的 $\alpha$-位上, 2010 年他们 ${ }^{[29]}$ 报道了在 $\mathrm{CuCl}$ 和有机小 分子催化剂存在下, 亲电三氟甲基化 Togni 试剂可不对 称地将三氟甲基引入到醛的 $\alpha$-位上(Scheme 10).

\section{2 我国学者在三氟甲基化反应的代表性工作}

\section{1 三氟甲基化试剂}

中国科学院上海有机化学研究所陈庆云教授等在 20 世纪 80 年代末至 90 年代相继发现七个对卤代烃的三 氟甲基化试剂 ${ }^{[4,30]}: \mathrm{FSO}_{2} \mathrm{CF}_{2} \mathrm{CO}_{2} \mathrm{Me}, \mathrm{ICF}_{2} \mathrm{SO}_{2} \mathrm{~F}$, $\mathrm{XCF}_{2} \mathrm{CO}_{2} \mathrm{Me}(\mathrm{X}=\mathrm{I}, \mathrm{Br}, \mathrm{Cl}), \mathrm{FO}_{2} \mathrm{SCF}_{2} \mathrm{CF}_{2} \mathrm{OCF}_{2} \mathrm{CO}_{2} \mathrm{Me}$, $\mathrm{FSO}_{2} \mathrm{CF}_{2} \mathrm{CF}_{2} \mathrm{OCF}_{2} \mathrm{CO}_{2} \mathrm{~K}$. 自 1989 年陈庆云教授等 ${ }^{[1]}$ 报 道 $\mathrm{FSO}_{2} \mathrm{CF}_{2} \mathrm{CO}_{2} \mathrm{Me}$ 在 $\mathrm{CuI}$ 的催化下可对芳基碘化物(烯 基溴和烯丙基溴化物)实现三氟甲基化反应后(Eq. 8), 该试剂已被国内外学术界和工业界广泛应用于合成含 三氟甲基的化合物 ${ }^{[4]}$.

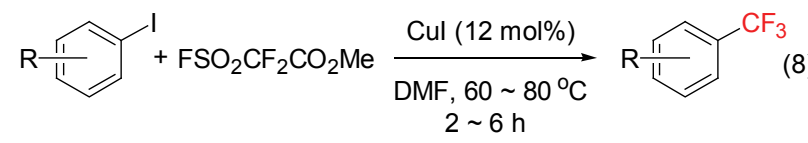

\section{2 钯催化的三氟甲基化反应}

中国科学院上海有机化学研究所刘国生教授等 ${ }^{[32]}$ 在探索钯催化形成 $\mathrm{C}-\mathrm{F}$ 的基础上, 2011 年他们 ${ }^{[33]}$ 报道 了钯催化下吲哚的氧化三氟甲基化反应. 在该反应中, 亲核性的三氟甲基化试剂 $\mathrm{CF}_{3} \mathrm{TMS}$ 作为三氟甲基源, $\mathrm{PhI}(\mathrm{OAc})_{2}$ 作为氧化剂, 为了抑制反应产生的三氟甲基 自由基对吲哚中苯环的进攻, 反应体系中加入 TEMPO. 最近刘国生教授课题组 ${ }^{[34]}$ 发现在钯催化下活泼烯烃的 氧化芳基三氟甲基化反应，该反应为合成含三氟甲基的 oxindoles 发展了新的方法(Scheme 11). 

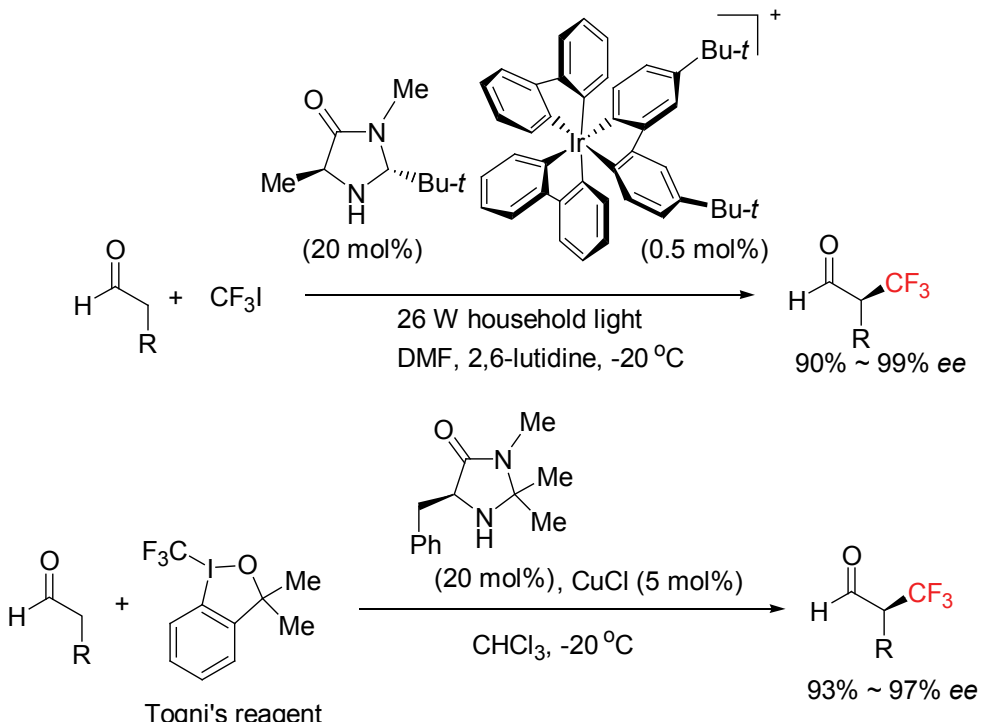

Scheme 10
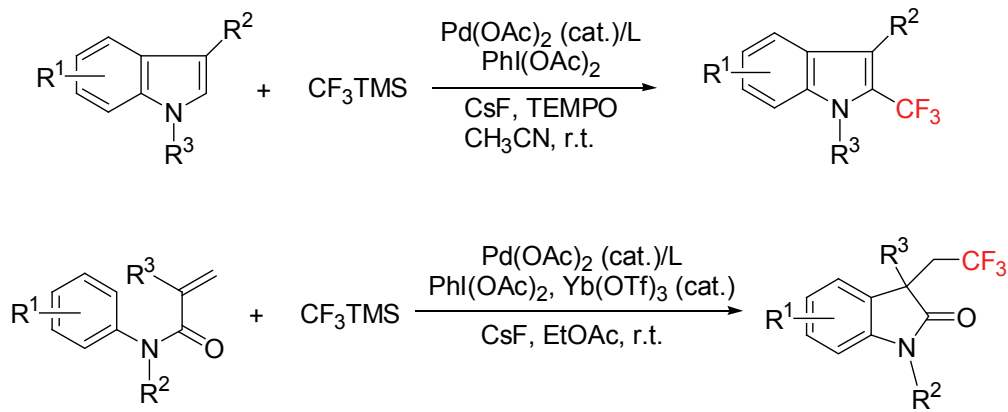

Scheme 11

最近中国科学院上海有机化学研究所胡金波教授 等 ${ }^{[35]}$ 采用空间位阻大的 Xantphos 为配体, 在 $80{ }^{\circ} \mathrm{C}$ 下实 现了钯催化芳基硼酸与三氟碘乙烷的偶联反应(Eq. 9), 为合成含三氟乙基的化合物发展了新的方法.

$$
\text { }
$$

\section{3 铜参与(催化)的三氟甲基化反应}

中国科学院上海有机化学研究所肖吉昌教授等发 现在铜粉存在下，亲电三氟甲基化试剂锍盐能够生成 $\mathrm{CF}_{3} \mathrm{Cu}$, 通过该反应现场生成的 $\mathrm{CF}_{3} \mathrm{Cu}$ 可与芳基杂环碘 化物反应高产率得到含三氟甲基的杂化化合物(Eq. $10)^{[36]}$.

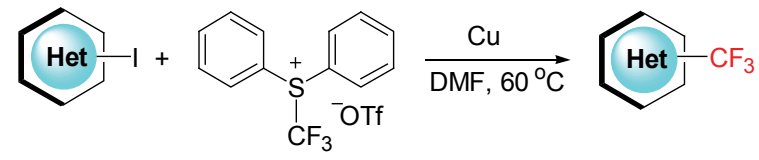

福州大学的翁自强教授等 ${ }^{[37]}$ 报道了 $\mathrm{AgF}$ 对铜催化 的三氟甲基化反应有协同作用. 在 $\mathrm{AgF}$ 存在下，吸电子 基团取代和推吸电子基团取代的芳基碘化物都能够顺 利地进行三氟甲基化反应(Eq. 11).

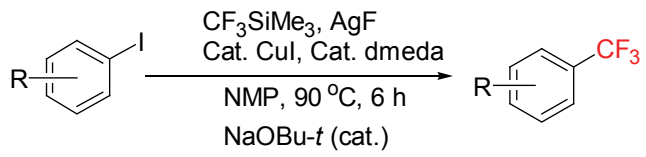

自 2010 年卿风领课题组开始探索芳基硼酸与亲核 三氟甲基化试剂的氧化三氟甲基化反应后，清华大学刘 否教授等 ${ }^{[38]}$ 、中国科学院上海有机化学研究所沈其龙教 授等 ${ }^{[39]}$ 和肖吉昌教授等 ${ }^{[00]}$ 分别开展了芳基硼酸与亲电 三氟甲基化试剂的反应研究(Scheme 12), 发展了反应 条件温和的三氟甲基化方法.

最近，沈其龙教授等 ${ }^{[41]}$ 报道了使用亲电三氟甲基 化试剂将三氟甲基引入到 1,3-二取代芳烃中. 该方法是 通过二步反应完成的: 首先 1,3-二取代芳烃在 Ir 的催化 下生成相应的芳基硼酸酯. 该芳基硼酸酯不需要分离纯 化, 然后在铜催化下与亲电三氟甲基化试剂反应得到三 
<smiles></smiles><smiles>[R1]c1ccc([Hg][14CH2]c2cccc3c2C(C)(C)OI3C(F)(F)F)cc1</smiles>

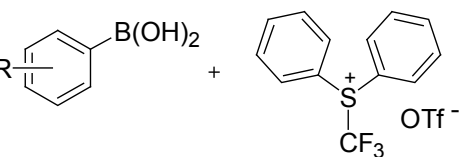
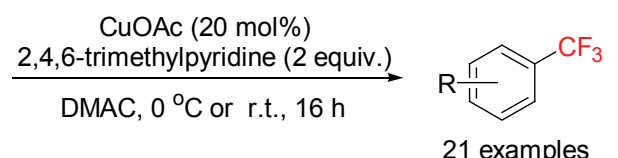

21 examples $40 \% \sim 78 \%$ yields

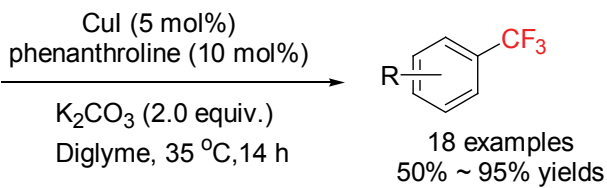

$\mathrm{Cu}$ (2 equiv.) $\mathrm{NaHCO}_{3}$ (1 equiv.)

DMF, $50^{\circ} \mathrm{C}, 11 \mathrm{~h}$

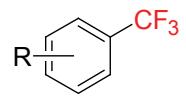

16 examples $46 \% \sim 75 \%$ yields

\section{Scheme 12}

氟甲基化合物(Eq. 12), 该方法可应用于向复杂分子的 芳环引入三氟甲基(如 Estrone 和 Vitamin $\mathrm{E}$ 等).

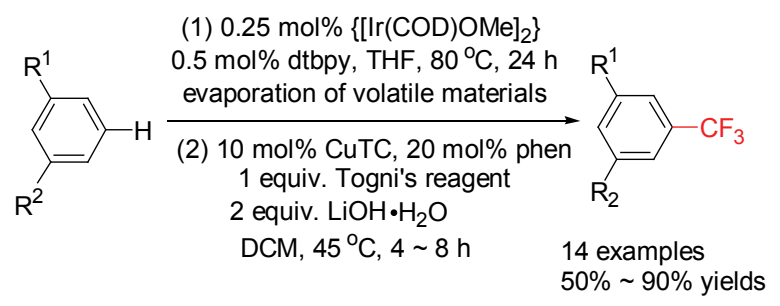

2011 年, 清华大学刘否教授 ${ }^{[42]}$ 和北京大学的王剑 波教授等 ${ }^{[43]}$ 发现在铜催化下, 亲电三氟甲基化试剂与 未活化的烯烃反应可生成三氟甲基化的烯丙基化合物 (Scheme 13), 他们对反应机理也进行了探索.

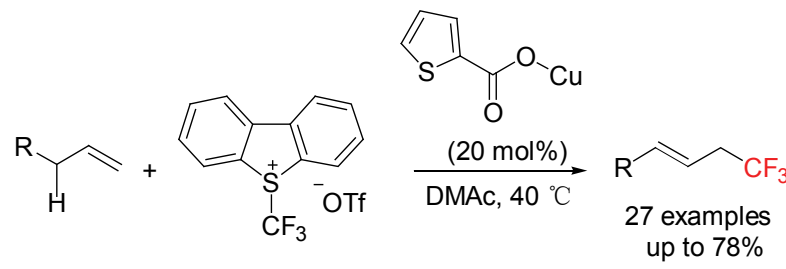

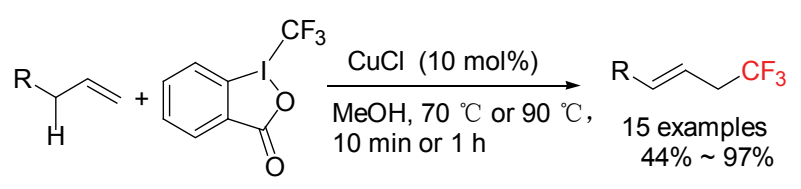

\section{Scheme 13}

\section{4 氧化三氟甲基化反应}

目前向有机分子引入三氟甲基的反应可分为如下 二大类反应(图 2): 亲电三氟甲基化试剂与亲核试剂的 反应; 但目前得到广泛应用的是含三氟甲基的亲核试剂 与相应的亲电试剂反应: 如芳基卤代物与当场产生的三
氟甲基铜 $\left(\mathrm{CuCF}_{3}\right.$ generated in situ $)$ 的偶联反应; 经三氟 甲基负离子(说明: 三氟甲基负离子不稳定，易分解. 到 目前为止，游离的三氟甲基负离子没有分离到)对羰基 类化合物的亲核加成反应等.

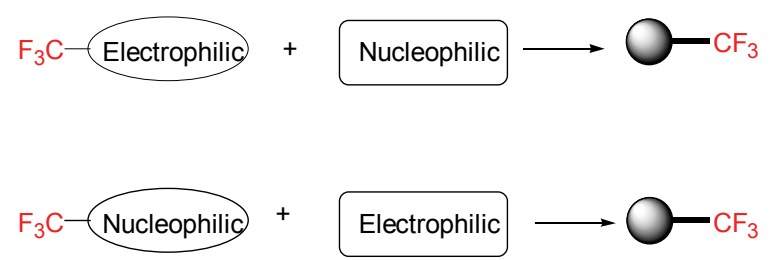

图 2 三氟甲基化反应

Figure 2 Trifluoromethylation

近几年来，过渡金属参与的亲核试剂之间经氧化形 成碳一碳键的偶联反应受到广泛关注，国际刊物发表了 一系列的研究成果. 在该研究方向结果的激发下, 卿凤 翎课题组提出了 “氧化三氟甲基化反应(oxidative trifluoromethylation)” 的新思想, 即在氧化剂存在下, 含三氟 甲基的亲核试剂与相应的亲核试剂反应(图 3). 如氧化 三氟甲基化反应能实现，将探索出新的三氟甲基化反应 类型; 同时也可发展 $\mathrm{C}-\mathrm{H}$ 键的直接三氟甲基化反应. 但由于三氟甲基基团的强吸电性, 要实现 “氧化三氟甲 基化反应”是一挑战性的课题.

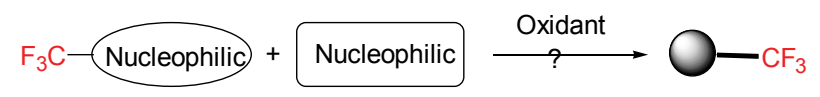

图 3 氧化三氟甲基化反应

Figure 3 Oxidative trifluoromethylation

2010 年, 卿凤领课题组 ${ }^{[44]}$ 实现了铜参与下的末端 炔烃与亲核三氟甲基化试剂 $\left(\mathrm{CF}_{3} \mathrm{SiMe}_{3}\right)$ 的氧化偶联反应 (Eq. 13). 利用空气作为氧化剂, 现场生成的高活性三氟 
甲基铜 $\left(\mathrm{CF}_{3} \mathrm{Cu}\right)$ 与末端炔烃反应生成三氟甲基炔类化合 物. 该反应首次实现了将三氟甲基直接引入到末端炔烃 的碳氢键上，为合成含三氟甲基的炔类化合物提供了简 便的方法. 实现该反应的关键是末端炔烃必须慢慢地加 入到现场生成的高活性的三氟甲基铜中, 否则得到的是 炔烃自身偶联产物. 另外, 配体 1,10-phenanthroline (phen)对稳定活性三氟甲基铜 $\left(\mathrm{CF}_{3} \mathrm{Cu}\right)$, 从而提高反应产 率起到重要作用.

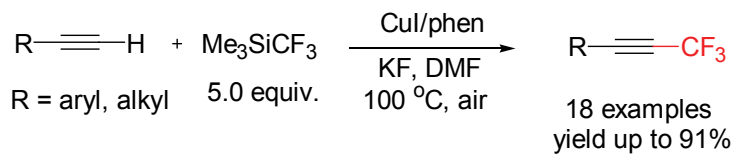

在以上研究的基础上, 卿风领课题组 ${ }^{[45]}$ 也实现了 铜参与下硼酸与亲核三氟甲基化试剂 $\left(\mathrm{CF}_{3} \mathrm{SiMe}_{3}\right)$ 的氧化 偶联反应(Eq. 14), 发展了一种选择性地向有机分子引 入三氟甲基的新方法. 该反应条件温和 $\left(45{ }^{\circ} \mathrm{C}\right.$ ), 芳基硼 酸和烯基硼酸都能高产率地转化为相应的三氟甲基化 合物. 更为突出的是: 羊基和溴取代的芳基嗍酸能生成 相应的三氟甲基芳基化合物，而羰基和溴在反应过程中 保持不变. 此研究结果发表后, Buchwald ${ }^{[26]}$, Hartwig ${ }^{[21]}$ 和 Gooßen 等 ${ }^{[25]}$ 进一步开展硼酸和硼酯的氧化三氟甲基 化反应; 刘否哊 ${ }^{[38}$ 、沈其龙 ${ }^{[39]}$ 和肖吉昌等 ${ }^{[40]}$ 研究了芳基硓 酸与亲电三氟甲基化试剂的反应.

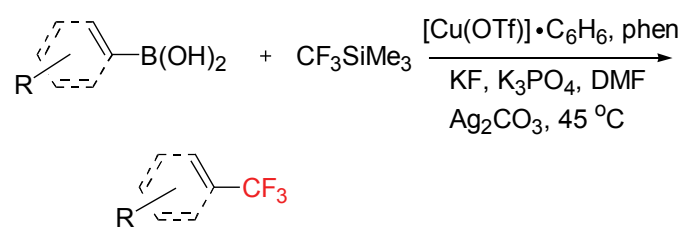

但以上二个反应(Eqs. 13 和 14)都有不足之处: 需要 当量的铜盐. 末端炔烃的氧化三氟甲基化体系需要 5 equiv. 的 $\mathrm{Me}_{3} \mathrm{SiCF}_{3}$ 和在 $100{ }^{\circ} \mathrm{C}$ 下反应(Eq. 13). 在 Hartwig 等 ${ }^{[20]}$ 报道 $\left[(\right.$ phen $\left.) \mathrm{CuCF}_{3}\right]$ 合成的启发下, 卿凤 翎课题组 ${ }^{[46]}$ 发现先原位生成较高活性和稳定性的 $\left[(\right.$ phen $\left.) \mathrm{CuCF}_{3}\right]$, 采用 $\mathrm{O}_{2}$ 作为氧化剂, 在室温下实现了末 端炔烃的三氟甲基化, 并且将 $\mathrm{Me}_{3} \mathrm{SiCF}_{3}$ 的用量降低到 了 2 equiv. (Eq. 15).

$$
\begin{aligned}
& \text { (1) phen (2.0 equiv.), } \mathrm{O}_{2}(101 \mathrm{kPa}) \\
& \mathrm{CuCl}+t \text {-BuOK } \underset{\mathrm{DMF}, 4 \mathrm{~A} \mathrm{MS} \text {, r.t., } 0.5 \mathrm{~h}}{(2) \mathrm{TMSCF}_{3}(2.0 \text { equiv. }) \text {, r.t., } 1 \mathrm{~h}} \\
& \text { (3) Terminal alkynes (1.0 equiv.) } \\
& 3 \text { h, r.t. } \\
& \mathrm{R}=\mathrm{CF}_{3}
\end{aligned}
$$

通过对反应机理的研究，从而改变 $\mathrm{Me}_{3} \mathrm{SiCF}_{3}$ 的进 样方式, 卿风领课题组 ${ }^{[47]}$ 成功地实现了铜催化下末端 炔烃和芳基硼酸的氧化三氟甲基化反应(Scheme 14).

卿风翎等 ${ }^{[48]}$ 也完成了在铜催化下一系列芳香杂环 和缺电子的多氟芳烃碳一氢键的直接氧化三氟甲基化 反应，不同类型的杂环使用不同的铜盐和氧化剂，该反 应为三氟甲基杂环化合物的合成提供了直接的方法 (Scheme 15).

2010 年, 卿凤领课题组 ${ }^{[49]}$ 报道了在氧化剂的存在 下，不需要金属催化剂，将三氟甲基引入到叔胺的 $\alpha$ 位(Eq. 16).

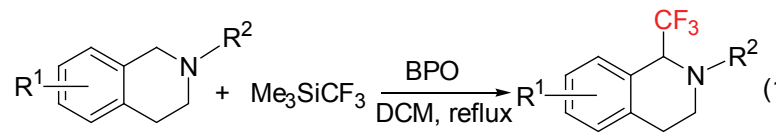

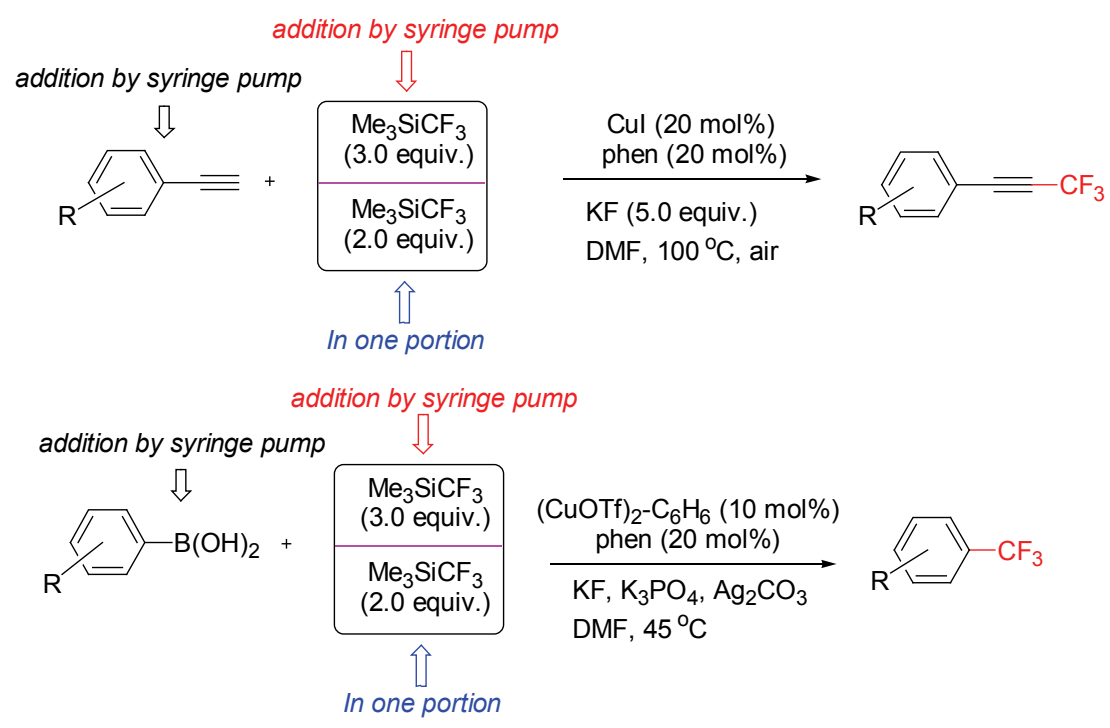

Scheme 14 

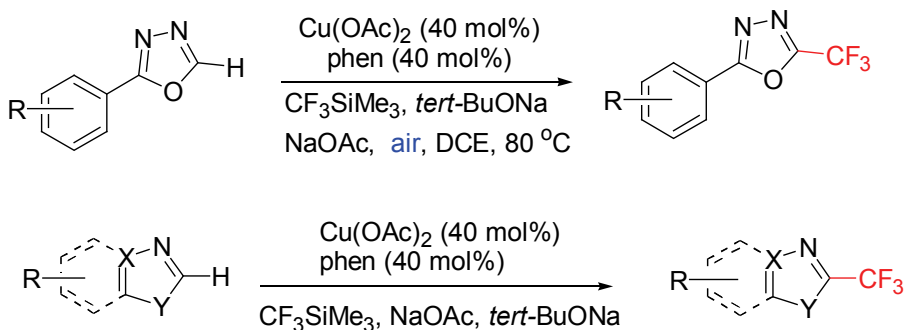

$\left(\mathrm{CH}_{3}\right)_{3} \mathrm{COOC}\left(\mathrm{CH}_{3}\right)_{3}$

DCE, $\mathrm{N}_{2}, 80^{\circ} \mathrm{C}$
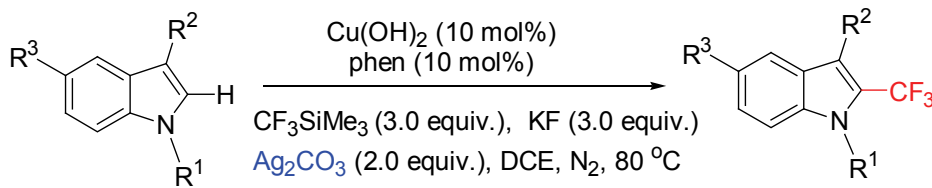

Scheme 15

最近, 卿风翎课题组 ${ }^{[50]}$ 将氧化三氟甲基化反应拓 展到氧化三氟甲硫基化反应 (oxidative trifluoromethylthiolation). 实现了铜催下硼酸与亲核三氟甲基化试剂 $\left(\mathrm{CF}_{3} \mathrm{SiMe}_{3}\right)$ 和硫粉三组分的氧化偶联反应, 发展了一种 向有机分子引入三氟甲硫基的新方法(Eq. 17). 该反应 条件温和操作简便: 把反应试剂加在一起, 在室温下反 应.

$$
\begin{aligned}
& \mathrm{CuSCN}(10 \mathrm{~mol} \%) \\
& \text { phen }(20 \mathrm{~mol} \%)
\end{aligned}
$$

\section{3 有机氟化学发展趋势}

学科本身的发展规律和社会需求推动学科的发展. 从有机氟化学的发展历史来看: 与其他学科比较, 社会 需求对有机氟化学的发展起到至关重要的作用. 笔者认 为有机氟化学应该会按照如下两点发展下去: 向有机分 子引入氟原子或含氟基团是有机氟化学研究永恒的课 题; 含氟生物活性物质和含氟功能材料是有机氟化学研 究的生命力之所在. 近年来有机氟化学研究取得的成果 和世界著名有机化学家涉足有机氟化学研究都说明有 机氟化学的发展是符合以上两点的. 笔者在此提出如下 一些值得关注的有机氟化学发展方向，供读者参考：

(1)尽管近年三氟甲基化反应取得重要进展, 三氟 甲基化反应仍将是有机氟化学的重要研究方向, 特别是 发展无金属存在下的三氟甲基化反应. 三氟甲氧基化反 应 $\left(\mathrm{CF}_{3} \mathrm{O}\right)$ 和三氟甲硫基化反应 $\left(\mathrm{CF}_{3} \mathrm{~S}\right)$ 将会引起更多的关 注.

(2) 由于大量的生物活性物质含有其他少氟基团
[如：一氟甲基 $\left(\mathrm{FCH}_{2}\right)$ 、二氟甲基 $\left(\mathrm{HCF}_{2}\right)$ 、二氟亚甲基 $\left(\mathrm{CF}_{2}\right)$ 、五氟化硫基 $\left(\mathrm{SF}_{5}\right)$ 等], 向有机分子引入这些少氟 基团的方法可借鉴三氟甲基化反应，但更重要的是发展 新型的氟化试剂及含少氟基团的合成子.

(3)含氟手性化合物在药物研发中起到重要作用, 因此发展不对称形成 C-F 键和 C- $\mathrm{CFXY}$ 的方法将是 有机氟化学的重要研究方向, 特别是向有机分子不对称 引入三氟甲基.

(4)发展反应条件温和、快速和高效形成 $\mathrm{C}-\mathrm{F}$ 的反 应，然后用于合成含 ${ }^{18} \mathrm{~F}$-标记的含氟化合物. PET(正电 子发射断层扫描技术)现已成为诊断和指导治疗肿瘤、 冠心病和脑部疾病这三大威胁人类生命的最优手段. 尽 管有许多可发射正电子的同位素可用于 PET 成像，但是 在临床学上 ${ }^{18} \mathrm{~F}$ 是最合适的放射性同位素. 因此含有 ${ }^{18} \mathrm{~F}$ 标记的化合物在 PET 成像中起到非常重要的作用 ${ }^{[1]}$. 由于 ${ }^{18} \mathrm{~F}$ 标记的化合物在医学上的重要性, 近几年将氟 原子引入芳环合成 $\mathrm{Ar}-\mathrm{F}$ 化合物是氟化学的研究热点 之一, 取得了一些重要进展 ${ }^{[52]}$.

(5)利用 $\mathrm{C}-\mathrm{F}$ 的特性和 ${ }^{19} \mathrm{~F}$ NMR 的高灵敏度及在生 命体内无干扰的特性, 开展有机催化剂、有机反应机理 及生命过程中的化学问题的研究.

(6)分子设计与合成含氟生物活性物质.

(7)设计与合成新型含氟单体, 研究含氟单体的聚 合反应规律，发展高新技术产业所需的特种含氟功能材 料.

致谢 感谢东华大学张柯同学和于锡闻同学为本文在 文献整理和部分反应式绘制做出的贡献.

\section{References}

[1] (a) Muller, K.; Faeh, C.; Diederich, F. Science 2007, 317, 1881. (b) Purser, S.; Moore, P. R.; Swallow, S.; Gouverneur, V. Chem. 
Soc. Rev. 2008, 37, 320

[2] Furuya, T.; Kamlet, A. S.; Ritter, T. Nature 2011, 473, 470.

[3] (a) Allemann, O.; Duttwyler, S.; Romanato, P.; Baldridge, K. K.; Siegel, J. S. Science 2011, 332, 574.

(b) Choi, J.; Wang, D. Y.; Kundu, S.; Choliy, Y.; Emge, T. J.; Krogh-Jespersen, K.; Goldman, A. Science 2011, 332, 1545.

(c) Lee, E.; Kamlet, A. S.; Powers, D. C.; Neumann, C. N.; Boursalian, G. B.; Furuya, T.; Choi, D. C.; Hooker, J. M.; Ritter, T. Science 2011, 334, 639.

(d) Rauniyar, V.; Lackner, A. D.; Hamilton, G. L.; Toste, F. D. Science 2011, 334, 1681.

[4] (a) Roy, S.; Gregg, B. T.; Gribble, G. W.; Le, V. D.; Roy, S. Tetrahedron 2011, 67, 2161.

(b) Tomashenko, O. A.; Grushin, V. V. Chem. Rev. 2011, 111, 4475.

[5] Ji, Y.; Brueckl, T.; Baxter, R. D.; Fujiwara, Y.; Seiple, I. B.; Su, S.; Blackmond, D. G.; Baran, P. S. Proc. Natl. Acad. Sci. U. S. A. 2011, 108, 14411.

[6] Langlois, B. R.; Laurent, E.; Roidot, N. Tetrahedron Lett. 1991, 32, 7525 .

[7] Nagib, D. A.; MacMillan, D. W. C. Natrue 2011, 480, 224.

[8] (a) Kamigata, N.; Fuukushima, T.; Yoshida, M. Chem. Lett. 1990, $19,649$.

(b) Huang, W. Y.; Lu, L. Chin. J. Chem. 1992, 10, 268.

[9] Culkin, D. A.; Hartwig, J. F. Organometallics 2004, 23, 3398.

[10] Grushin, V. V.; Marshall, W. J. J. Am. Chem. Soc. 2006, 128, 12644.

[11] Watson, D. A.; Su, M.; Teverovskiy, G.; Zhang, Y.; GarcíaFortanet, J.; Kinzel, T.; Buchwald, S. L. Science 2009, 325, 1661.

[12] Cho, E. J.; Senecal, T. D.; Kinzel, T.; Zhang, Y.; Watson, D. A.; Buchwald, S. L. Science 2010, 328, 1679.

[13] Cho, E. J.; Buchwald, S. L. Org. Lett, 2011, 13, 6552.

[14] (a) Ball, N. D.; Kampf, J. W.; Sanford, M. S. J. Am. Chem. Soc. 2010, 132, 2878.

(b) Ye, Y.; Ball, N. D.; Kampf, J. W.; Sanford, M. S. J. Am. Chem. Soc. 2010, 132, 16482.

(c) Ball, N. D.; Gary, J. B.; Ye, Y.; Sanford, M. S. J. Am. Chem. Soc. 2011, 133, 7577.

[15] Wang, X.; Truesdale, L.; Yu, J. Q. J. Am. Chem. Soc. 2010, 132, 3648 .

[16] McLaughlin, V. C. R.; Thrower, J. Tetrahedron 1969, 25, 5921.

[17] Kobayashi, Y.; Kumadaki, I. Tetrahedron Lett. 1969, 10, 4095.

[18] Wiemers, D. M.; Burton, D. J. J. Am. Chem. Soc. 1986, 108, 832.

[19] Dubinina, G. G.; Furutachi, H.; Vicic, D. A. J. Am. Chem. Soc. 2008, 130, 8600 .

[20] Morimoto, H.; Tsubogo, T.; Litvinas, N. D.; Hartwig, J. F. Angew. Chem., Int. Ed. 2011, 50, 3793.

[21] Litvinas, N. D.; Fier, P. S.; Hartwig, J. F. Angew. Chem., Int. Ed. 2012, 51, 536 .

[22] Tomashenko, O. A.; Escudero-Adán, E. C.; Belmonte, M. M.;
Grushin, V. V. Angew. Chem., Int. Ed. 2011, 50, 7655.

[23] Oishi, M.; Kondo, H.; Amii, H. Chem. Commun. 2009, 1909.

[24] Knauber, T.; Arikan, F.; Roschenthaler, G.-V.; Gooßen, L. J. Chem. Eur. J. 2011, 17, 2689.

[25] Khan, B. A.; Buba, A. E.; Gooßen, L. J. Chem. Eur. J. 2012, 18, 1577.

[26] Sencal, T. D.; Parsons, A. T.; Buchwald, S. L. J. Org. Chem. 2011, 76, 1174.

[27] Parson, A. T.; Buchwald, S. L. Angew. Chem., Int. Ed. 2011, 50, 9120.

[28] Nagib, D. A.; Scott. M. E.; MacMillan, D. W. C. J. Am. Chem. Soc. 2009, 131, 10875 .

[29] Allen, A. E.; MacMillan, D. W. C. J. Am. Chem. Soc. 2010, 132, 4986.

[30] Chen, Q. Y. J. Fluorine Chem. 1995, 72, 241.

[31] Chen, Q. Y.; Wu, S. W. J. Chem. Soc., Chem. Commun. 1989, 705.

[32] Qiu, S.; Xu, T.; Zhou, J.; Guo, Y.; Liu, G. J. Am. Chem. Soc. 2010, $132,2856$.

[33] Mu, X.; Chen, S.; Zhen, X.; Liu, G. Chem. Eur. J. 2011, 17, 6039.

[34] Mu, X.; Wu, T.; Wang, H. Y.; Guo, Y.; Liu, G. J. Am. Chem. Soc. 2012, 134, 878 .

[35] Zhao, Y.; Hu, J. Angew. Chem., Int. Ed. 2012, 51, 1033.

[36] Zhang, C. P.; Wang, Z. L.; Chen, Q. Y.; Zhang, C. T.; Gu. Y. C. Xiao, J. C. Angew. Chem., Int. Ed. 2011, 50, 1896.

[37] Weng, Z.; Lee, R.; Jia, W.; Yuan, Y.; Wang, W.; Feng, X.; Huang, K. W. Organometallics 2011, 30, 3229.

[38] Xu, J.; Luo, D. F.; Xiao, B.; Liu, Z. J.; Gong, T. J.; Fu, Y.; Liu, L. Chem. Commun. 2011, 4300.

[39] Liu, T.; Shen, Q. Org. Lett. 2011, 13, 2342

[40] Zhang, C. P.; Cai, J.; Zhou, C. B.; Wang, X. P.; Zheng, X.; Gu, Y. C.; Xiao, J. C. Chem. Commun. 2011, 47, 9516.

[41] Liu, T.; Shao, X.; Wu, Y.; Shen, Q. Angew. Chem., Int. Ed. 2012, $51,555$.

[42] Xu, J.; Fu, Y.; Luo, D. F.; Jiang, Y. Y.; Xiao, B.; Liu, Z. J.; Gong, T. J.; Liu. L. J. Am. Chem. Soc. 2011, 133, 15300.

[43] Wang, X.; Ye, Y.; Zhang, S.; Feng, J.; Xu, Y.; Zhang, Y.; Wang, J. J. Am. Chem. Soc. 2011, 133, 16410.

[44] Chu, L.; Qing, F. L. J. Am. Chem. Soc. 2010, 132, 7262.

[45] Chu, L.; Qing, F. L. Org. Lett. 2010, 12, 5060.

[46] Zhang, K.; Qiu, X. L.; Huang, Y.; Qing, F. L. Eur. J. Org. Chem. 2012, 58 .

[47] Jiang, X.; Chu, L.; Qing, F. L. J. Org. Chem. 2012, 77, 1251.

[48] Chu, L.; Qing, F. L. J. Am. Chem. Soc. 2012, 134, 1298.

[49] Chu, L.; Qing, F. L. Chem. Commun. 2010, 46, 6285.

[50] Chen, C.; Xie, Y.; Chu, L.; Wang, R. W.; Zhang, X.; Qing, F. L. Angew. Chem., Int. Ed. 2012, 51, 2492.

[51] (a) Cai, L.; Lu, S.; Pike, V. W. Eur. J. Org. Chem. 2008, 2853. (b) Littich, R.; Scott, P. J. H. Angew. Chem., Int. Ed. 2012, 51, 1106.

[52] Furuya, T.; Klein, J. E. M. N.; Ritter, T. Synthesis 2010, 1804.

(Zhao, X.) 